\title{
EL VOCABULARIO DE LA ORIENTACIÓN EN EL USO DE LA LENGUA EN LOS DICCIONARIOS DE LINGÜÍSTICA
}

\section{LANGUAGE USAGE VOCABULARY IN LINGUISTICS DICTIONARIES}

\author{
Félix Valentín Bugueño Miranda \\ Universidade Federal do Rio Grande do Sul (UFRGS). Brasil \\ felixv@uol.com.br
}

\section{Resumen:}

En el variado ámbito de perspectivas de la lingüística, pareciera que el espacio reservado a la orientación en el uso de la lengua ocupa un lugar secundario. Uno de los vehículos que refleja estas perspectivas es el diccionario de lingüística. En este contexto el objetivo del presente trabajo es investigar los posibles vocablos referentes a la orientación en el uso de la lengua presentes en esta clase de obras de referencia. Como metodología se trabajará con un axioma que permitirá detectar los vocablos pertinentes a la investigación. Los resultados demuestran que el abanico de vocablos es numéricamente extenso y variado. Como conclusión es posible afirmar que la orientación en el uso de la lengua es un fenómeno vivamente presente en la discusión lingüística, especialmente en diccionarios de lingüística redactados por lingüistas franceses.

Palabras clave: Orientación; norma, prescripción; uso

\begin{abstract}
:
Among the multiple perspectives of linguistics, it seems to be that language correction has a secondary importance. Some perspectives of linguistics can be observed in reference works such as dictionaries of linguistics. This paper aims to search for words that refer to language correction in this kind of reference work. As a methodological framework we use an axiom to detect such words. Our results show that there is a wide number and range of words related to language correction. It is possible to contend that the vocabulary of language correctness is still present in the linguistic discussion, especially in dictionaries of linguistics produced by French linguists.
\end{abstract}

Keywords: language guidance; norm; prescription; usage.

Recibido: 24 de junio de 2019

Aceptado: 15 de diciembre de 2019

A la memoria de mi padre, Félix Segundo Bugueño Toro ( 2019), quien me enseñó lo que era un «lapsus linguae» y un «lapsus calami».

\section{Introducción}

Baldinger plantea la existencia de tres planos en una lengua natural: la lengua objeto, que corresponde a la actividad lingüística propiamente tal; el metalenguaje de 
primer nivel, que corresponde a la función metalingüística de Jackobson (126), o sea, la posibilidad de convertir a la lengua en objeto o referente de la actividad lingüística y, finalmente, el metalenguaje de segundo nivel, que corresponde a la metodología de la lingüística.

Al ámbito del metalenguaje de primer nivel se adscribe un fenómeno propio de la actividad del hablar como lo son las language beliefs, esto es, las creencias sobre el lenguaje. No obstante, la profusión de conceptos y designaciones que la literatura ofrece (cf., por ejemplo, Abdi y Asidi (106)), se puede definir creencias sobre el lenguaje como todo aquello que los miembros de una comunidad idiomática conjeturan y/o perciben en relación con su lengua materna u otra(s) lengua(s).

Un ejemplo de estas creencias es la posición de relieve (lengua global) que ocuparía el inglés (cf., por ejemplo, Crystal English, Nunan ${ }^{1}$ ). Otra manifestación es la creencia de que hay variedades del español, por ejemplo, que se demostrarían claramente inferiores a otras (cf., por ejemplo, Morales Pettorino) $)^{2}$.

Lo expuesto por Morales Pettorino está intrínsecamente relacionado al fenómeno de la sanción idiomática, esto es, la determinación de lo que está correcto e incorrecto, respectivamente. Sin embargo, Rabanales advirtió hace ya más de treinta años que esta dicotomía es insuficiente. Es por ello que al hacer cualquier afirmación sobre el uso de la lengua es más prudente hablar de orientación en el uso de la lengua. Esta orientación obedece a lo que Zanatta denomina ansia normativa [anseio normativo], esto es, la búsqueda por una forma preferencial ante la duda idiomática. Elizancín lo ha formulado de una manera acertadamente plástica: "El ciudadano común quiere saber si tal frase es correcta o no. Y quiere que las academias se expidan sobre ello, sin ambigüedad, dejando de lado el relativismo imperante [...]”.

La lingüística moderna ha sentido una desconfianza casi generalizada frente a estas dos categorías, aunque particularmente frente a la primera, llamada también de prescripción. No cabe la menor duda de que tal posición se deriva, evidentemente, de

\footnotetext{
${ }^{1}$ En cuanto Crystal English y Nunan exponen panoramas sobre la expansión del inglés y su consolidación de lengua allende cualquier frontera, Moreno Fernández argumenta exactamente en sentido contrario, ofreciendo una interesante exposición de por qué ni el inglés ni ninguna lengua natural podría llegar nunca a constituir una lengua global.

${ }^{2}$ El fenómeno de las creencias conoce otras dimensiones más. Así, por ejemplo, en el caso específico de Brasil, la expansión de la enseñanza del español a partir de la última década del siglo XX despertó expectativas con relación a que el dominio de tal lengua iba a permitir insertarse mejor en el mercado de trabajo o que su enseñanza ofrecería mejores resultados en términos de rendimiento escolar, ya que esta lengua sería supuestamente más fácil que otras lenguas extranjeras (cf. Zolin-Vesz). A este ámbito corresponde también la utilización de lo que Boysson-Bardies (232) llama de manierismos, esto es, el uso de eufemismos para atender así a lo "políticamente correcto".
} 
una de las máximas saussureanas ${ }^{3}$ que enfatiza que la tarea del lingüista es describir los hechos lingüísticos, no juzgarlos. Así, la oposición prescripción / descripción se ha convertido en una verdadera antinomia (cf. Kirchner et (s.v. Antinomie)), o como lo describe Crystal The Cambridge (p.3), "la oposición ha sido presentada en términos casi políticos de liberalismo radical frente a conservadurismo elitista"4. De forma complementaria, cabe también tener en mente lo expresado por Elizaincín, quien distingue entre una "lingüística sin adjetivos" (de clara inspiración saussureana) y una lingüística que se preocupa de la relación entre las lenguas "y el individuo que las usa y con la sociedad que las alberga". Es en el seno de esta segunda lingüística que tienen acogida los language beliefs.

Es evidente que en un escenario donde se enfrentan un liberalismo radical y un conservadurismo elitista - para emplear las palabras de Crystal The Cambridge-, entreverado a su vez por las distinciones de Elizaincín, parece no haber espacio en los repertorios de vocablos ${ }^{5}$ que explicitan los conceptos propios de las ciencias del lenguaje para un subconjunto de términos relacionados a la orientación en el uso de la lengua.

\section{Los diccionarios de lingüística}

En el ámbito de los repertorios léxicos que se restringen a determinados ejes del diasistema de una lengua natural, el diccionario de lingüística ocupa un lugar sui generis. Por un lado, desde el punto de vista del diasistema, estas obras se restringen al eje diatécnico. Por otro lado, su objetivo es ofrecer una serie (normalmente extensa) de afirmaciones sobre una lengua natural considerada ya en el nivel del metalenguaje. Por tanto, no es exagerado afirmar que un diccionario de lingüística constituye el metalenguaje del metalenguaje. Al referir cómo un determinado término opera en el seno de una teoría lingüística o de un nivel de organización de la lengua, los diccionarios de lingüística corresponden al metalenguaje de segundo nivel, esto es, a la metodología de la lingüística. No obstante que es posible definir el diccionario de lingüística tanto en relación con qué eje del diasistema se restringe, así como también al tipo de predicación que presenta, es sintomático que no encuentre ni siquiera una

\footnotetext{
${ }^{3}$ Cf., por ejemplo, CLG $(12,109$ y 144) con una clara posición a favor de la tarea descriptiva de la lingüística.

4 [The opposition has been presented in quasi-political terms - of radical liberalism vs elitist conservatism]

5 Se emplea la expresión vocablo, pues no hay certeza de que lo codificado en los diccionarios de lingüística corresponda a términos, tal como los entiende la terminología.
} 
mínima mención en los propios diccionarios de lexicografía, como es el caso de Hartmann y James y Martínez de Souza.

En relación con la selección lemática que hagan, uno de los rasgos arquetípicos de esta clase de obras es su (a veces) abierta filiación a determinadas "escuelas" de la lingüística, así como su ocasional (e insoslayable) silencio frente a otras corrientes de las ciencias del lenguaje. Así, por ejemplo, el Dictionnaire de Linguistique et des Sciences du Langage (DlaL) es prolijo en artículos referentes al análisis del discurso (analyse de discours, langagier, etc.) y al funcionalismo francés, pero prácticamente ignora la lingüística del texto. De la misma forma, un término proveniente del idealismo alemán, sentimiento lingüístico [Sprachgefühl], no aparece lematizado ni en el Dictionary of Linguistics and Phonetics (DLP) ni en el Oxford Concise Dictionary of Linguistics (OxDL).

Ad supra se ha señalado que la orientación en el uso de la lengua ha sufrido una general deslegitimación como materia o tópico propio de la investigación y reflexión sobre el lenguaje. De la misma forma, se ha destacado que los diccionarios de lingüística demuestran una, a veces, insoslayable adhesión a determinadas escuelas de la lingüística, hecho que pareciera señalar que, hipotéticamente al menos, no habría cabida en aquellas obras para ningún término que denotara el ámbito de la orientación en el uso de la lengua.

Con el objetivo de confirmar (o refutar) esta hipótesis, este trabajo consistirá en la formulación de una clasificación para constatar y evaluar si un conjunto de diccionarios de lingüística da cabida a términos referentes a la orientación en el uso de la lengua.

\section{Procedimientos metodológicos}

Desde una perspectiva metodológica, la primera cuestión a ser resuelta es establecer lo que con alguna propiedad podría denominarse un corpus glossariorum scientiarum linguae. La cuestión no es menor si se considera que las designaciones empleadas en los títulos de tales diccionarios no son siempre convergentes. Así, frente a designaciones por equivalentes, tales como diccionario de lingüística, linguistics dictionary, dictionnaire de linguistique y Sprachwissenschaftliches Wörterbuch, hay otras menos evidentes, como lo son Terminologie zur neueren Linguistik, Dictionnaire de Lexicologie Française, o Diccionario de términos filológicos. En relación con este segundo grupo, la expresión Terminologie sugiere que se trataría de un glosario con claros fines de fijación de una lengua de especialidad. La Terminologie zur neueren 
Linguistik (Abraham) corresponde a un diccionario de lingüística con un recorte claramente sincrónico (del estructuralismo al generativismo con alguna apertura a la lingüística del texto). En relación con Dictionnaire de Lexicologie Française, y no obstante que, de facto, hay una ingente cantidad de términos de esta área de estudios del lenguaje, esta obra constituye también un diccionario de lingüística. Finalmente, la denominación términos filológicos evoca un énfasis en la diacronía de la lengua y en la fijación y exégesis de textos; el título Diccionario de términos filológicos (Lázaro Carreter) corresponde también a un diccionario de lingüística, aunque, evidentemente, se da generosa cabida a las áreas antes mencionadas.

La selección del corpus glossariorum scientiarum linguae se realizó atendiendo a dos criterios: en primer lugar, que correspondiera a obras editadas en los últimos veinte años. Esta cesura obedece al hecho de que la edición de diccionarios de lingüística no es particularmente frecuente. En segundo lugar, no hay cómo negar que las tradiciones de estudios del lenguaje de lengua inglesa, francesa y alemana son las que más aportan en términos cuantitativos y cualitativos. Con ello no se está negando en modo alguno lo que se publique en otras lenguas y/o provenga de otros espacios geográficos ${ }^{6}$. En función de lo expuesto, el corpus está compuesto por las siguientes obras: el Oxford Dictionary of Linguistics (OxDL) y el Dictionary of Linguistics and Phonetics (DLP) para la lengua inglesa, el Dictionnaire Larousse de Linguistique et des Sciences du Langage (DLaL) y el Dictionnaire de Lexicologie Française (DLexFr) para la lengua francesa, el Metzler Lekikon Sprache (MLSp) y el Routledge Dictionary of Language and Linguistics (DLL) 7 para la lengua alemana.

En relación con los criterios de búsqueda, se torna necesaria la formulación de un axioma $^{8}$ que permita la exploración de posibles vocablos en los diccionarios citados en el párrafo anterior. Para ello, parece apropiado crear primeramente un marco teórico que sirva como referencia para la elaboración de dicho axioma, lo que permitirá identificar los vocablos referentes a la orientación en el uso de la lengua.

\footnotetext{
${ }^{6}$ En el caso de la lengua española, por ejemplo, Lázaro Carreter y Alcaraz Varó y Martínez Linares son obras notables. Sin embargo, la tercera edición de Lázaro Carreter no aporta mucho en relación a la segunda. En lo que respecta a Alcaraz Varó y Martínez Linares, hay una edición de 2004 que está agotada y a la que no fue posible acceder. La mayoría de las obras de esta naturaleza que están disponibles en la actualidad son reimpresiones de ediciones antiguas. Los textos escogidos para el análisis corresponden a ediciones actualizadas.

${ }^{7}$ DLL (2006) es una traducción al inglés del Lexikon der Sprachwissensschaft (Stuttgart: Alfred Kröner Verlag). No obstante, el título en inglés de la edición que se usará para este trabajo se trata, en realidad, de un diccionario alemán de lingǘstica.

${ }^{8}$ Axioma es una aserción que se asume como base para fines de investigación. Cf. Blackburn (s.v. axioma).
} 
Coseriu distingue entre norma real y norma ideal. La primera corresponde a la actividad lingüística efectivamente realizada por una comunidad de hablantes (equivale al ergon de Humboldt). De forma concomitante a esa manifestación del hablar, una comunidad de hablantes distingue entre las múltiples manifestaciones del hablar efectivamente realizado (entre las varias normas reales) una que le sirva de modelo u orientación. A esta modalidad se la denomina norma ideal. En función de ello, la comunidad compara entre lo que es la actividad real y el modelo que le sirve de orientación. La confrontación para distinguir lo correcto de lo incorrecto o -si se quierela desviación entre una realización lingüística concreta y un modelo era ya un tópico entre los griegos, quienes estaban preocupados con la etimología, en la medida en que la reflexión etimológica les permitía restituirles a las palabras su forma original, modificada por el uso (cf. Robins (23). No cabe duda de que un hito en la historia de la orientación en el uso de la lengua es la doctrina del bon usage, claramente prescriptiva; todo lo que no esté en consonancia con este bon usage será tildado de mal uso [mauvais usage]. De esta forma, la orientación se entiende como una auténtica dicotomía: lo correcto frente a lo incorrecto.

No obstante, un hecho ontológico del lenguaje es la variación, tal como Coseriu lo expone a través del concepto de diasistema, esto es, una colección de lenguas funcionales. Por ello, la noción dicotómica de correcto frente a incorrecto debe ser necesariamente ampliada para correcto (frente a incorrecto) y ejemplar, para usar la terminología empleada por Moreno de Alba. La cuestión se torna así más compleja, en la medida que el patrón de evaluación se desplaza del monocentrismo al pluricentrismo, como Farías y Maldonado Cárdenas lo reclaman para una lengua como el español, por ejemplo. La cuestión no es menor si se piensa que la orientación y la sanción en el uso de la lengua adquieren un tinte casi político en la medida que desembocan en lo que se denomina como políticas y planificación lingüísticas (cf. Wee para un panorama sobre esta dimensión) ${ }^{9}$. De esta forma, habrá que asumir que factores extralingüísticos terminan formando parte de la orientación en el uso de la lengua también.

$\mathrm{Si}$, por un lado, la estructura de una lengua ha sido concebida en relación con el conjunto de lenguas parciales (normas) que inherentemente la conforman, no es menos

\footnotetext{
${ }^{9}$ Según Wee (11), la política y la planificación lingüística en los continentes asiático y africano corresponden a la pugna en países descolonizados entre el deseo de una norma propia y el apego de ciertos sectores a la norma de la potencia colonizadora. La síntesis crítica que el autor hace sobre estas políticas es más bien pesimista. No obstante que este tópico no forma parte de este trabajo, no hay cómo negar que razón tiene Crystal The Cambridge cuando advierte que la corrección en el uso de la lengua acaba impregnándose de una dimensión extralingüística, política.
} 
verdadero que una lengua está constituida también por niveles de organización (Henne). Para efectos de este trabajo, se considerarán los niveles fónico, ortográfico, morfológico, sintáctico y léxico. La orientación en el uso de la lengua se torna concreta en cada uno de ellos en la medida que existe para cada nivel de organización un conjunto de vocablos para señalar un fenómeno que precisa de atención.

A partir de las consideraciones precedentes, se buscará en los diccionarios de lingüística todo vocablo que responda al siguiente axioma: "Cualquier vocablo que denote lo que constituye una aserción, una recomendación o una censura en relación con el uso de la lengua".

\section{Adendas al axioma:}

1. El vocablo puede ser de proyección vertical u horizontal. Esto quiere decir que la orientación acontece no sólo en relación con el eje diafásico-diastrático, sino que también al eje diatópico ${ }^{10}$.

2. El vocablo puede estar referido a los niveles de organización de la lengua fónico, ortográfico, morfológico, sintáctico y léxico.

3. El vocablo puede referirse a expresiones referenciales (nombres propios) o expresiones múltiples o de referencia dividida (nombres comunes) ${ }^{11}$.

A partir del axioma y sus adendas, se encontraron los siguientes conjuntos de artículos lexicográficos ${ }^{12}$ :

\section{OxDL:}

adaptation " $[\ldots]$ process by which loan words are changed to fit the sound patterns of the language into which they are borrowed."

argot "Special vocabulary used e.g. by criminals [...]."

bleaching " Change by which the meaning of a word becomes increasingly unspecific",

Descriptive (1) "[...] Also opposed to *prescriptive [...]."

euphemism "Word, etc. used in place of one avoided as e.g. offensive, indecent, or alarming [...]."

language planning "Any attempt by a government, etc. to favour one language, or one form of language, over another."

normative "(Grammar, rule) seeking to establish or prescribe usage. [...]."

prescriptive (1) "(Grammar, rule) which aims to 'prescribe' what is judged to be correct rather than to 'describe' actual usage [...]."

proscribe "To condemn as e.g. contrary to a rule."

\footnotetext{
${ }^{10}$ La distinción no es menor si se considera que explica, por ejemplo, la precisión hecha en Moreno de Alba en relación con el español. Además, refleja también la postura axiológica del francés frente al inglés.

${ }^{11}$ Para estas precisiones se han seguido las distinciones propuestas por Zimmermann y Sternfeld (62-64).

${ }^{12}$ Debido a la limitación de espacio, se transcribirán únicamente trechos de cada artículo.
} 
DLP:

archaism "A term used in relation to any domain of language structure for an old word or phrase no longer in general spoken or written use."

avoidance languages " $[\ldots]$ a term used to characterize languages which permit communication between a person and others with whom there is a social taboo." grammar "[...](1) A descriptive grammar is, in the first instance, a systematic description [...](4) The phrase traditional grammar [...]is usually used with a critical ('non-scientific') implication [...]. Criticism is directed primarily at the prescriptive and proscriptive recommendations of authors [...]."

hypercorrection "[...] The phenomenon usually takes place when speakers of a non-standard dialect attempt to use the standard dialect and 'go too far' [...]."

norm "[...] term $[\ldots]$ to refer to a standard practice in speech or writing $[\ldots] . "$

orthoepy, "[...] an old term (dating from the seventeenth century) for the study of correct pronunciation and of the relationship between pronunciation and the writing system."

prescriptive "A term used by linguists to characterize any approach which attempts to lay down rules of correctness as to how language should be used", proscriptive, "A term used by linguists to characterize any approach which attempts to lay down rules of correctness, emphasizing how language should not be used."

purism "A term used pejoratively in linguistics to characterize a school of thought which sees a language as needing preservation from the external processes which might infiltrate it and thus make it change [...]."

received pronunciation "The name given to the regionally neutral accent in British English, historically deriving from the prestige speech of the Court and the public schools. The term indicates that its prestige is the result of social factors, not linguistic ones."

standard, "A term used in sociolinguistics to refer to a prestige variety of language used within a speech community [...]."

standard English "In sociolinguistics, a much-debated term for the variety of English used as a communicative norm throughout the English-speaking world. The notion has become increasingly difficult to handle because of the emergence of differing national standards of usage [...]."

usage "The collective term for the speech and writing habits of a community, especially as they are presented descriptively with information about preferences for alternative linguistic forms. Linguists emphasize the importance of describing the facts of usage as a control on the claims made by grammars and contrast this emphasis with the prescriptive attitudes of traditional grammar, whose rules often bore no relationship to what people actually did with their language."

\section{DLaL:}

abus "[...] marques de rejet qui signalent les sens ou les mots rejetés par les puristes [...]."

archaïsme "[...] une forme lexicale ou une construction syntaxique appartenant $[\ldots]$ à un systéme disparu $[\ldots] . "$

argot "[...] dialecte social réduit au lexique de caractère parasite [...]"

attitude "2. Les attitudes langagières constituent l'ensemble des opinions explicites ou implicites sur l'usage d'une langue."

autorité "[...] quelqu'un [...] quand une communauté socioculturelle lui reconnaît le droit de définir ce qui est à dire et ce qui n'est pas à dire [...]."

barbarisme "[...] forme d'un mot qui n'est pas conforme aux règles de la langue [...]."

bon usage "La norme, ensemble de règles qu'il faut respecter pour bien parler la langue [...]."

cacologie "[...] construction syntaxique agrammaticale [...]."

cacophonie "[...] répétition, jugée désagréable à entendre, des mêmes sons [...]."

classique "[...] état de langue [...] pris comme référence et norme [...]."

correction "[...]. On dit qu'un énoncé est correct, quand il est conforme non seulement à la grammaire de la langue, mais aux règles du «bien-dire» fixées par un group social généralement réduit, mais socialement dominant."

dirigisme " Le dirigisme linguistique est l'attitude des autorités politiques qui réglementent les usages d'une langue, ou, dans le cas de plurilinguisme, interviennent sur le status des langues en présence." 
franglais "Le franglais désigne d'une manière pejorative l'ensemble des néologismes d'origine angloaméricaine introduits dans la langue française depuis les années 50."

hypercorrection "[..] il y a hypercorrection, quand, en présence d'une forme altérée par l'évolution de la langue, on restitue une forme où les éléments que l'on croit disparus sont ajoutés par erreur."

libéralisme glottopolitique "[...] est l'attitude fondée sur le principe du «laissez faire», [...] ne pas intervenir pour ou contre une norme, une variété ou une langue, et à laisser agir les tendences dominantes."

néologisme "[...] unité lexicale [...] qui n'était pas réalisée antérieurement."

normalisé "Une langue est dite normalisé quand 1'action des autorités de planification visent à en écarter les irrégularités ou ce qui est considéré comme tel."

normatif "La grammaire s'est parfoit réduite à une série de préceptes normatifs, c'est-à-dire à une série d'instructions qui finalement se résolvent à dites $X$, ne dites pas $Y$."

normativisation " $[. .$.$] tendance à imposer une norme, c'est-à-dire un ensemble de prescriptions sur les$ variantes linguistiques qu'on doit employer au détriment d'autres."

norme "[...] système d'instructions définissant ce qui doit être choisi parmi les usages d'une langue donée si l'on veut se conformer à un certain idéal esthétique ou socioculturel. [...] implique l'existence d'usages prohibés [...]."

orthoépie "[...] science qui définit la prononciation correcte d'un phonème [...]."

orthographe "Le concept de orthographe implique la reconnaisance d'une norme écrite par rapport à laquelle on juge l'adécuation des formes [...]."

orthophonie "[...] la prononciation considérée comme correcte et normal d'un phonème ou d'une suite de phonèmes (mots)."

planification linguistique "Ensemble de mesures ordonées prises para un État pour la normalisation d'une langue ou de son emploi."

plurinormaliste "[...] tout attitude qui admet dans une langue l'éxistence de plusieurs normes [...] comme tout également dignes d'attention."

pureté “[...] la qualité d'un usage de la langue qui serait exempt de toute contamination étrangère ou populaire."

purisme "Comportement de certains locuteurs vis-à-vis de leur langue, caracterisé par le désir de fixer celle-ci à un stade de son évolution consideré comme una norme idéale et intangible [...]."

solécisme "[...] une construction de phrase qui n'est pas produite par les régles de la grammaire d'une langue à une époque déterminée ou bien qui n'est pas acceptée dans une norme ou un usage jugé correct." trivial "En lexicographie [...] marque stylistique attribuée à des termes qui appartiennent à divers nivaux de langue [...] et qui sont condamnés par les contraintes socioculturelles parce qui íls dénotent des objets jugés «indécents» ou expriment des attitudes «grossières» ou « obscènes»."

usage "l'ensemble des règles de grammaire relativement stabilisées et utilisées par le plus grand nombre de locuteurs [...]."

Vaugelas “[...] Ses remarques sur la langue française [...] ont pour but de donner le «bon usage», celui de l'élite sociale et intellectuelle."

xénisme "[...] unité lexicale constituée par un mot d'une langue étrangère et désignant une realité propre à la culture des locuteurs [...]."

DLFr:

Abusif "Les dictionnaires d'usage indiquent parfois que tel emploi d'un mot est «abusif», ou «impropre», ou «critiqué», ce qui veut dire le plus souvent que le mot est alors consideréé comme étant employé dans un sens qui n'est pas tout à fait le sien."

Académie Française " [...] a recu par mission fixer la langue française, de lui donner des règles à respecter [...]."

Acceptable "Pour qu'un nouveau mot [...] soit acceptable, il ne suffit pas que la nouveauté ainsi créée soit «bien formée», c'est-à-dire conforme aux règles et contraintes de la lexicogenèse dans un état de langue $[\ldots] . "$

À-la-modisme, ou alamodisme "Mot, expression ou locution en vogue, dont l'emploi, courant dans la presse parlée et écrite, est teintée de snobisme [...]."

Alexical "[...] Se dit d'un mot qui ne serait pas « bien formé », du fait qu'il ne respectareit pas les règles lexicogéniques, [...]. Un mot alexical est aussi désigné sous le nom de barbarisme." 
Anglomanie "Manie qu'ont certains usagers snobs et sublettrés d'employer des mots anglais ou pseudoanglais dans un énoncé, qui est censé être en français."

Argot "[...] les usagers actuels utilisent le mot argot dans un sense étendu et considèrent aussi comme « de l'argot » les éléments appartenant aux niveaux de langue populaire et vulgaire de la langue commune $[\ldots] . "$

Attraction paronymique "Autrefoi désignée sou le nom de contamination (ce qui implique une vision prescriptive [=normative] et non descriptive [...]."

Autocensure lexicographique "Les auterurs et éditeurs de dictionnaires ont longtamps exclu de la nomencalture de ces ouvrages des sens, des mots et des locutions frappés d'un tabou dans la societé française [...] concernant sourtout les organes et les fonctions d'excrétion et de la reproduction (sexualité) et certains jurons"

Barbarisme voir à Alexical

Bien formé "Une lexie ets bien formée quand elle est conforme aux règles et contraintes lexicales en usage dans un état de langue [...]."

Catachrêse "La catachrèse n'est pas l'emploi «abusif» ou «incorrect» d'un mot, comme certains lexicographes prescriptivistes le prétendent, mais l'extension d'emploi d'un mot qui est admise par l'usage [...]."

Cliché "Lexie, séquence ou phrase tout faite plus ou moins imaginée, caractérisée par sa banalité et sa grande fréquence d'emploi [...]."

Descriptif, "[...] Ainsi, on substitue le descriptif au prescriptif [...]."

Dysphémisme "[...] le dysphémisme [...] implique une volonté de choisir una façon plus brutale de s'exprimer, souvant teintée d'une certaine méchanceté sarcastique."

Erreur "La forme et le sens de certaines lexies peuvent être dus historiquement à des erreurs ou confusions de diverse nature."

Faux emprunt "La méconnaissance de l'anglais ainsi que le snobisme anglomane et l'américanolâtrie, qui l'accompagnent curieusement, notamment parmi les professionels des médias, plus spécialement les journalistes «sportifs», sont à l'origine de la création et de la diffusion de formations pseudo-anglaises ou faux emprunts."

Normatif "[...] préceptes dits normatifs parce qu'ils se fondaient sur une norme établie d'après «le bon usage» et «les bons auteurs», sur les bases non pas linguistiques mais socio-culturelles, la langue fixée comme référence étant en fait celle des milieux socio-professionnels priviligiés."

Norme "Tout ce qui [...] est d'usage courant dans une communauté constitue la norme linguistique."

Orthographe "Manière d'écrire un mot que l'on considère comme étant la seule «correcte»."

Pédantisme "Lexie, tl'emploi révèle chez son utlilsateur une volonté de faire un étalage d'érudition ou de distinction."

Péjorativ "On dit qu'un mot, ou une locution, est péjoratif [...] lorsqu'il implique un jugement défavorable sur la qualité du référent [...]."

Prescriptif voir normatif; voir aussi puriste

Puriste ou [...] purisme "Un puriste est une personne de mentalité prescriptiviste (= normative) qui prétend défendre sa langue maternelle contre tout ce qui est susceptible d'en affecter la «pureté» [...]. En matière de lexique, le purisme se justifie beaucoup moins qu'en grammaire."

Raciste "Quelques dictionnaires signalent certains mots comme ayant une connotation injurieuse ou raciste."

Recommandé "Le Journal officiel peut recommander d'utiliser tel ou tel mot français existent [...]."

Usage "[...] on appelle usage la manière dont est utilisé par les locuteurs natifs, dans un état de langue donné, l'ensemble [...] des lexies [...] réalisées et des règles et contraintes qu'on peut en induire."

MLSp:

Allgemeiner Deutscher Sprachverein “ [...] Ziel des Vereins war «die Reinigung der deutschen Sprache von unnöthigen fremden Bestandteile» im allgemeinen vaterländisch-nationalist. Rahmen.”

Amtssprache "[...] die für den amtl. Verkehr zugelassene Spr. [...]."

Archaismus "Wörter, Wortformen, syntakt. Erscheinungen und Schreibungen, die für einen best. Zeitraum als veraltend gelten und schlie $\beta 1$. als veraltet betrachtet werden."

Bildungssprache "Sprachformen, die überwiegend von Bevölkerungsgruppen mit hohem Bildungsniveau verwendet werden [...]."

Dummdeutsch "Titel zweier sprachkrit. Wörtersammlungen, gerichtet gegen die «schleichende Verhunzung unserer Sprache» [...], in denen auffällig dumme Neuprägungen und Neuverwendungen von Wörtern $[\ldots]$ gesammelt $[\ldots]$ werden" 
Gemeinsprache "Erscheinungsform einer Spr., die im gesamten Sprachgebiet als verbindl. Vorbild für alle Sprachteilnehmer gilt [...]."

Gesellschaft für deutsche Sprache "[...] erteilt prakt. Auskünfte in sprachl. Zweifelsfällen [...]."

Hochsprache "[...] verwendeter Ausdruck, der auf die höhere Entwicklungsstufe (im Vegleich zu Dialekten und Umgangssprachen) [...] überwiegend auf die höheren Sozialschichten verweisst."

Hyperkorrektur "Sprachform, die im übermäßigen Bemühungen um Korrektheit falsch wurde."

Kanzleisprache "Geschriebene Sprache der größeren städl. und fürstl. bes. kaiserl. Kanzleien in frühnhd. Zeit [...]."

Kauderwelsch "[...] (welsch « romanisch» i.S. von « nicht deutsch») Adj. [...] mit der Bedeutung «unverständlich, fremdklingendes, gegen Sprachnormen verstoßender Sprachgebrauch»."

Kunstwort "Bewußt gebildetes Wort zur Bez. von neuen (meist wiss. oder tech.) Begriffen sowie Warennamen für die neue oder neu angebotene Produkte [...]."

Nationalsprache "[...] Gelegentlich im Sinne von Standardvarietät oder nationaler Amtssprache eines Staates."

Neologismus "Eine durch Neubildung oder Entlehnung neu entstandene lexik. Einheit [...]."

normativ "Zumeist Gegenbegriff von deskriptiv, bezogen auf die Absicht oder die Wirkung der Tätigkeit von Sprachwissenschaftlern bei der Standarisierung von Spr. [...]."

Normative Grammatik "Grammatik, die Regeln und Normen aufstellt um eine Hochsprache zu konservieren [...]."

Normtoleranz "[...] Einstellung (Attitude), die Abweichungen von den eigenen oder von der in der jeweiligen Situation ansonsten geltenden Norm duldet [...].”

Orthographie "[...] bezeichnet einmal die Gesamtheit der (amtl.) normierten Schreibkonventionen [...]."

Orthographiereform "[...] Ihr Ziel ist es, dem Schreiber die Anwendungen der Orhographie leichter zu machen, ohne dabei dem Leser die Entschlüßelung zu erschweren."

Orthographisches Wörterbuch "Ein notwendiger, aber kein hinreichender Grund für spezielle o. Wbb, ist die mangelnde Vorhersagberkeit der Schreibung einzelner Wörter [...]."

Plastikwörter "[...] Traktat, [...] das eine Reihe von Substantiven, die im öffentl. Diskursen hochfrequent sind, als referenzarm und gleichzeitig prestigträchtig geißelt."

Plurizentrische Sprache "Spr. mit mehreren nat. Standardvarietäten [...]."

Prestige "Ansehen, das ein bestimmtes Individuum aufgrund bestimmter Attribute oder das ein bestimmtes Attribut in einer Gesellschaft hat [...] auch eine Sprech/Schreibweise oder Sprachform [...]. Ggs. Stigma [...]"

Rotwelsch "[...] Sondersprachen von Teilen der untersten Sozialschichten in der Fuktion von Geheimsprachen [...]."

Solözismus “[...] Bez. für Verstöße gegen korrekten Sprachgebrauch, v.a. in Bezug auf die Syntax [...].” Sprachenpolitik "[...] S. richtet sich auf das Verhältnis zwischen verschiedenen Spr.[...]."

Sprachgebrauch "Der Gebrauch der Sprache wie der eines Wortes wird in der Philosophie der normalen Sprache durch die soziale Verwendungsweise in Situationskontexten, nicht aber durch die Angabe einer Menge von Grbrauchsinstanzen bestimmt"

Sprachkritik "Auseinandersetzung und Beurteilung herrschender Sprachnormen, von Stilkritik über die Kritik von Sprachformen der Presse, des Rundfunks und Fernsehens"

Sprachnorm "Das sprach. Korrekte, Richtige."

Sprachnormung "Plannug und Festlegung fachsprachlichen Termini oder von Standardvarietäten [...]."

Sprachpolitik "Im Gegensatz zu Sprachenpolitik auf eine einzlne Sprache gerichtet [...]. S. versucht v.a., durch Verbot oder Vorschrift bestimmter Wörter und Wendungen das Bewusstsein der Sprecher zu beeinflussen [...]."

Tautazismus "Abwertender Begriff aus der normativen Stilistik, der »Verstöße« gegen den lautl. »Wohlklang« bezeichnet [...]."

DLL:

archaism „The effective use of outdated expressions for poetic, ironic, or elevated connotation.”

barbarism "A term in classical rhetoric for the improper use of a word. [...] barbarism was later used for mistakes in orthography, pronunciation, and agreement $[\ldots]$ (solecism)."

cacophony "Linguistic or musical discord resulting from a disagreeable combination of sounds. The antonym is euphemism."

cliché "Pejorative term taken from printers' language, generally used to refer to a commonly occurring utterance that is used schematically."

euphemism "An agreeable combination of sounds." 
hypercorrection "Process and result of an exaggerated attempt on the part of a speaker to adopt or imitate linguistic forms or a linguistic variety that he/ she considers to be particularly prestigious."

language policy "Political measures aimed at introducing, implementing, and defining the regional use of languages, such as the use of individual languages in multilingual states (language planning)."

language regulation " 1 In the narrow sense, involvement in the use of language (usually by the state) aimed at bringing about or suppressing certain conscious associations. (language manipulation, language planning, language policy) 2 In the broad sense, any kind of intentional control of language use, often (though not necessarily exclusively) with a view to affect-ing the denotation and connotation of certain terms, by any group with a vested interest."

linguistic criticism "[...] (b) evaluation of linguistic norms (e.g. elaborate vs restricted code [...]."

linguistic norms "Social expectations, which determine the forms of suitable linguistic interaction within the boundaries of the linguistic system."

neologism "Newly formed linguistic expression (word or phrase) that is recognized by at least part if not all of a language community as the way to denote a new object or state of affairs."

pedagogical grammar "Grammatical textbook or handbook for students or teachers of language that presents the grammar (usually prescriptive grammar) in a pedagogically based approach."

pejorative "Semantic characteristic of linguistic expressions which invoke negative connotations: such derogatory meaning components can be created by new formations (e.g. wet-backs for 'illegal Mexican immigrants'), by meaning change, (e.g. dame, originally '(noble)lady'), as well as by prefixes such as mal-, pseudo-."

prescriptive grammar "Form of grammatical description with the goal of instruction in the proper use of language and which is influenced by historical, logical and aesthetic considerations. [...] prescriptive grammars lag behind the development of theoretical, or descriptive grammars."

school grammar "[...] Its general characteristics are: [...] (c) primarily a prescriptive attitude ( prescriptive grammar) i.e. concerned with judgments such as 'correct,' 'incorrect,' 'affected,' 'awkward' [...]."

solecism "A term from rhetoric for an infraction of the rules of grammar. Solecism, like barbarism, affects the principle of correctness of language, which is the first of the four qualities of style in classical rhetoric."

taboo word "A term that is avoided for religious, political, or sexual reasons and is usually replaced by a euphemism, e.g. rest room or bathroom for toilet."

Los resultados de la búsqueda demuestran que los diccionarios de lingüística acogen un conjunto bastante variado y extenso de vocablos referentes a la orientación idiomática. Es sintomático que no sólo recogen un repertorio léxico de expresiones metalingüísticas, sino que también hasta nombres propios relacionados a la actividad de orientación. Además, la muestra compilada revela también que la dimensión de la orientación posee límites difusos en algunos casos, siendo a veces difícil establecer de manera clara la pertinencia de un vocablo dentro del esquema de clasificación ${ }^{13}$. El esquema de clasificación propuesto es de orden taxonómico. El esquema establece una distinción entre una dimensión lingüística (las palabras que se refieren a palabras) y otra extralingüística (las palabras que se refieren a las cosas) ${ }^{14}$. En el seno de la distinción lingüística opera una oposición entre lo indicativo y lo exhortativo. Lo indicativo se refiere a cualquier aserción respecto de un hecho idiomático referido a la orientación,

\footnotetext{
${ }^{13}$ Sobre esto ver la observación de Glück y Sauer más adelante en el cuerpo del trabajo.

${ }^{14}$ Para esta distinción, téngase presente lo expuesto en la nota 11. Sobre este particular, Engelberg y Lemnitzer (14) destacan la dificultad de separar el conocimiento lingüístico [sprachliches Wissen] del conocimiento enciclopédico [enzyklopädisches Wissen]. Esta dificultad queda muy bien representada por el artículo lexicográfico Dummdeutsch en MLSp (2010, s.v.).
} 
pero sin juzgarlo. Lo exhortativo, por otra parte, se refiere a vocablos de función axiológica. Se reconoce, dentro de este plano, una oposición entre lo que se preceptúa y lo que se veda.

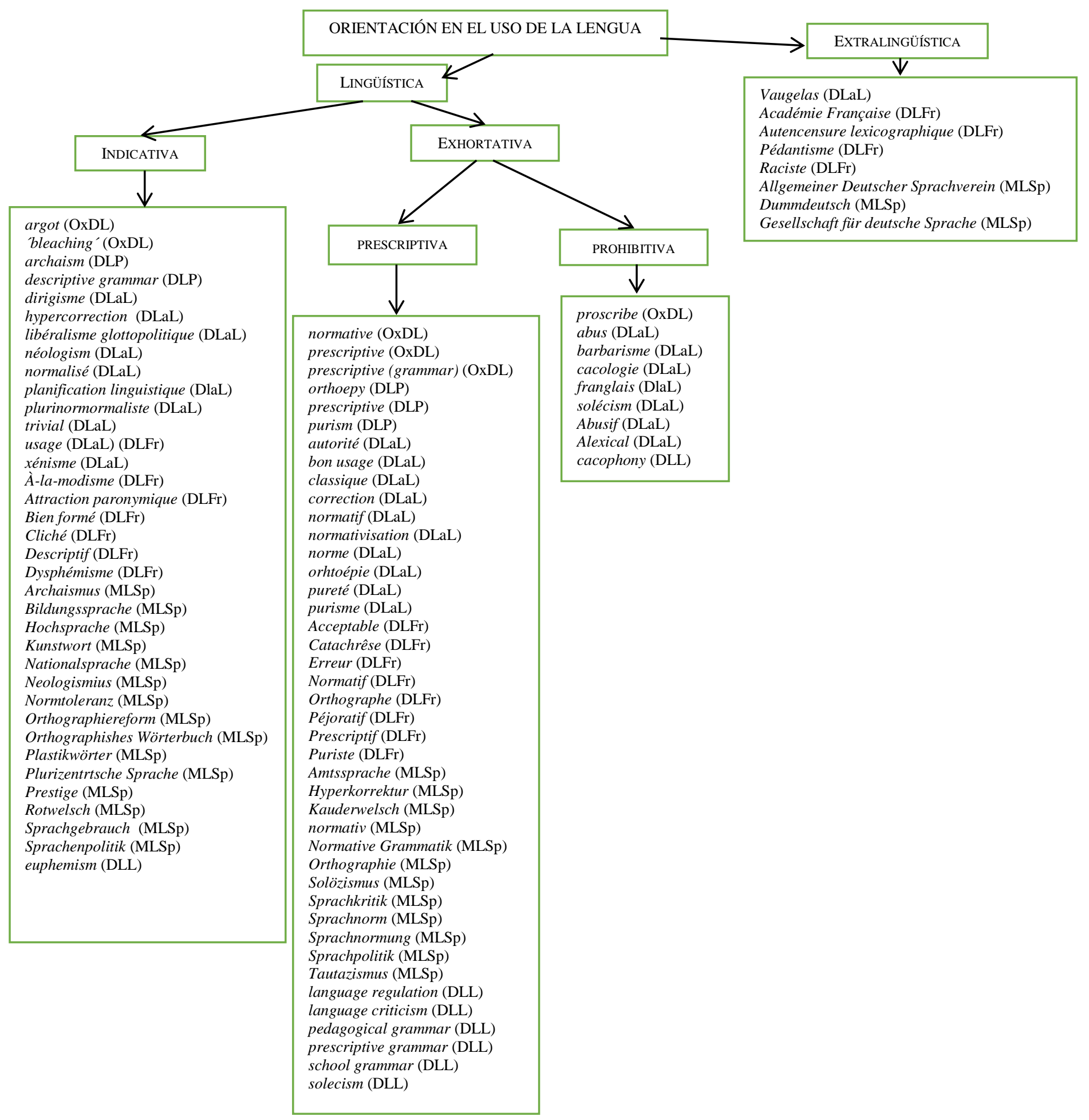


El análisis del inventario léxico compilado y evaluado torna posible llegar a un conjunto variado de conclusiones. En primer lugar, la compilación de vocablos de cada diccionario analizado permite constatar que los diccionarios de lingüística ingleses son los que menos compilan léxico referente a la orientación en el uso de la lengua. Al otro extremo se encuentran los diccionarios de lingüística de lengua francesa. En un punto intermedio, los del alemán. No cabe duda de que los extremos que representan los diccionarios del inglés y del francés están en directa relación con el pensamiento lingüístico del que estos diccionarios son un reflejo. Por un lado, la lingüística inglesa se ha debatido entre la prescripción y la descripción. Peters (765), por ejemplo, menciona que una evaluación de veinte guías de uso demostró que la tendencia prescriptiva era acentuada en estas obras. Al mismo tiempo, no hay cómo negar que la investigación empírica, basada en corpus (propiciada por Sinclair, por ejemplo), ha desplazado la perspectiva de análisis a favor de la descripción. Sintomáticamente, el diccionario constituye el catalizador de esta disputa, que perceptiblemente se inclina a favor de esta última tendencia, como lo comprueba, por ejemplo, la discusión de Mugglestone (70-112) ${ }^{15}$. Por otro lado, en el francés se advierte una tendencia claramente prescriptiva, anclada en la doctrina del bon usage, y que en los días de hoy se manifiesta en lo que Eckkrammer y Lescure (134) denominan planificación lingüística [aménagement linguistique], que no es otra cosa que la constante intervención del Estado para mantener la lengua ajena a fuerzas externas y preservarla de perder su legitimidad ${ }^{16}$. En lo que respecta al alemán, su posición entre los extremos corresponde al equilibrio entre la lingüística moderna (del estructuralismo en adelante) y la filología ${ }^{17}$. Dos manifestaciones de este equilibrio son el diccionario ortográfico y el diccionario de extranjerismos, que ofrecen orientaciones sobre ortografía y el

\footnotetext{
${ }^{15}$ Esta discusión, sin embargo, no parece completamente justa, ya que, para probar el carácter falaz de la prescripción en la lengua inglesa, Mugglestone emplea ejemplos de diccionarios de estadios muy anteriores de la lengua.

${ }^{16}$ Sobre este particular, Walter (329-330) divide la responsabilidad del cuidado de la lengua entre lo que denomina los verdaderos árbitros del uso [les vrais arbitres de l'usage], que no son otros que Le Petit Robert y Le Petit Larousse (por la pérdida de legitimidad que experimentó Le Dictionnaire de l’Académie), y la intervención del Estado, como comentado ad supra.

${ }^{17}$ Se advierte, sin embargo, una tendencia a posiciones que se acercan a la prescripción. Kunkel-Razum, por ejemplo, constituye un intento por reavivar la discusión en torno a la observancia de la ortografía. De facto, el título del trabajo, Por qué no da lo mismo que escribamos de cualquier manera [Warum es nicht egal ist, wie wir schreiben] constituye una ardua defensa de la prescripción en este nivel de organización de la lengua.
} 
significado de las unidades exógenas a la lengua ${ }^{18}$. En última instancia, no hay cómo negar que estas perspectivas a veces tan disímiles revelan ideologías lingüísticas, esto es, el conjunto de creencias, sentimientos y concepciones que se tenga sobre una lengua, como las define Piller (4), y en las que se cruzan factores tan heterogéneos como el bon usage y el advenimiento de la linguística de corpus ${ }^{19}$. Ad infra se mencionarán vocablos rotulados de idiosincráticos. Estos vocablos son ejemplos palpables de ideología lingüística.

Un segundo aspecto para considerar es la distribución de los vocablos en la taxonomía. En términos numéricos los $\operatorname{taxa}^{20}$ establecidos ofrecen los siguientes resultados: 37 vocablos de orden Indicativo, 42 vocablos de orden Prescriptivo, 8 vocablos de orden Prohibitivo; el $\operatorname{taxon}^{21}$ Extralingüística, a su vez, presenta un total de 8 vocablos. Es claramente perceptible una diferencia mínima entre lo que constituye lo indicativo en relación con determinados fenómenos del lenguaje y lo que es prescriptivo. Evidentemente que estos resultados hay que evaluarlos a la luz de la contribución masiva de vocablos de raigambre prescriptiva aportados por los diccionarios del francés. No obstante, pareciera, prima facie, que la lingüística, particularmente la anglosajona, se debate entre el ansia normativa de los hablantes (Zanatta) y el prurito de describir, pero no juzgar ${ }^{22}$. Es notorio que Peters (765) se apoye en un total de veinte guías de uso para hacer el análisis en su investigación. Los guías de uso existen justamente porque el hablante vacila frente a la lengua. En el párrafo anterior se ha mencionado también a Mugglestone y su opción por la descripción. Sus observaciones sobre la función sancionadora deficiente de parte de la lexicografía inglesa permiten entrever también que su postura deja espacio para una moderada orientación en el uso de la lengua con la condición de que esta orientación se fundamente en evidencia empírica.

En este mismo ámbito es notorio el bajo número de vocablos que proscriben un determinado uso o forma. Su bajo número se debe muy probablemente a aquello que

\footnotetext{
${ }^{18}$ Esta clase de diccionarios cuenta con muchos exponentes en el seno de la tradición lexicográfica alemana. Ello se debe a que la comunidad de hablantes de esa lengua, a diferencia de la francesa, presenta una tolerancia frente a los elementos léxicos exógenos a la lengua.

${ }^{19}$ No cabe duda de que las ideologías lingüísticas juegan un papel central en el tópico expuesto aquí. Por una cuestión de espacio, no es posible extenderse más sobre este particular.

${ }^{20}$ Para el concepto de taxa, cf Marradi.

21 Para el concepto de taxon, cf. Marradi.

${ }^{22}$ Glück y Sauer (26) sostienen que, no obstante que hay buenas razones para actuar con moderación y ceñirse únicamente al ámbito de la descripción, el problema no se resuelve simplemente por el hecho de que un hecho lingüístico se evalúa a la luz de la objetividad científica.
} 
Crystal The Cambridge (3) expone de manera magistral, esto es, el embate entre un supuesto conservadurismo y un progresismo lingüístico. El vocabulario referente a la orientación idiomática refleja lo que son las tendencias de la lingüística, pero da pocas pistas sobre el origen del ámbito descriptivo, prescriptivo y prohibitivo. En lo que se refiera a la antinomia descriptivo / prescriptivo-prohibitivo, no cabe duda de que parte del rechazo a este segundo miembro de la antinomia está relacionado también a una tendencia de las obras de orientación, que es la de sancionar ex nhilo, o sea, sin que las decisiones cuenten con un respaldo teórico metodológico ${ }^{23}$.

Como tercer aspecto cabe destacar que no existe siempre una convergencia conceptual entre determinados vocablos. Así, por ejemplo, la definición de norm en DLP (s.v.) corresponde a lo que Coseriu denomina norma real, en cuanto que la definición de norme en DLaL (2013, s.v.) corresponde a la norma ideal. Otro tanto acontece con purism, DLP (s.v.) y purisme, DLaL, (s.v.) y DLFr, (s.v.). Esta discrepancia se explica por la adopción de una postura descriptiva en la lingüística inglesa en oposición a una postura prescriptiva de la lingüística francesa, como ya se comentó en los párrafos anteriores.

Un cuarto aspecto son los casos de convergencia parcial en la significación, tales como argot OxDL (s.v.), DLaL (s.v.), DLFr (s.v.) y Rotwelsch MLSp (s.v.); néologisme DLaL (s.v.), Neologismus, MLSp (s.v.) y neologism DLL (s.v.); hypercorrection, DLP (s.v.), hypercorrection, DLaL (s.v.), Hyperkorrektur, MLSp (s.v.) y hypercorrection, DLL (s.v.). Llama la atención que la convergencia fonológico-ortográfica de estos vocablos en las distintas lenguas (con la única excepción de Rotwelsch) permitiría hacer pensar que se trata de internacionalismos $y$, por ende, se esperaría también una convergencia en el plano del significado. No fue posible encontrar un patrón que permitiera explicar esta discrepancia parcial en el plano del contenido.

En relación con vocablos tales como los tratados en los dos últimos párrafos ad supra, Glück y Sauer (23) comentan que sería una tarea estéril ofrecer definiciones apropiadas [griffige Definitionen] para tales palabras, esto es, definiciones convergentes y exactas, ya que en su formulación intervienen parámetros muy diferentes, tales como sociológicos, psicológicos, etc.

\footnotetext{
${ }^{23}$ Un ejemplo es NDDúvP. No obstante que la metalexicografía de lengua portuguesa no forma parte de este trabajo, NDDúvP es un caso muy claro de esta tendencia.
} 
Un quinto aspecto son los muchos casos de convergencia total, tales como solécisme, DLaL (s.v.), Solözismus, MLSp (s.v.) y solecism, DLL (s.v.); normative, OxDL (s.v.), normatif, DLaL (s.v.), DLFr (2017, s.v.) y normativ, MLSp (s.v.) ${ }^{24}$.

El análisis constató también la presencia de nombres propios. En este sentido la orientación parece cumplir un rol determinante en el seno de algunas comunidades lingüísticas en las que las academias, individuos y/o obras representan per se la orientación en el uso de la lengua, como lo son los casos de Académie Française, DLFr (s.v.), Allgemeiner Deutscher Sprachverein, MLSp (s.v.)), Dummdeutsch, MLSp, (s.v.), Gesellschaft für deutsche Sprache, MLSp (s.v.) e Vaugelas, DLFr (s.v.).

Un aspecto que tampoco puede dejar de mencionarse es lo que se podría denominar de idiosincrático, esto es, lo propio de una determinada cultura lingüística, que se manifiesta en términos como Autencensure lexicographique, DLFr (s.v.), bon usage, DLaL (s.v.), Dummdeutsch, MLSp (s.v.), Faux emprunt, DLFr (s.v.), franglais, DLaL (s.v.), Kanzleisprache, MLSp (s.v.), Kauderwelsch, MLSp (s.v.), Pédantisme, DLFr (s.v.), pureté, DLaL (s.v.) y Raciste, DLFr (s.v.). En el caso de los vocablos franceses, es insoslayable el rechazo a las influencias de la lengua inglesa. A ello súmase el papel central que ocupa el diccionario en la orientación en el uso de la lengua, que trasciende lo estrictamente lingüístico, como es el caso de Autocensure lexicographique, Pédantisme e pureté. En el caso del alemán, la etimología de Kauderwelsch ("no románico"), revela los residuos de una xenofobia lingüística.

En términos generales, la gama de conceptos y designaciones refleja el amplio espectro de posiciones sobre la orientación en el uso de la lengua. Prima facie, pareciera que la lingüística está frente a una verdadera aporía, una inviabilidad racional. No obstante, un análisis más ponderado permite comprender que la dimensión de la orientación es multifacética per se. En este sentido, la primera lección es comprender finalmente que la orientación no es ni puede ser dicotómica. Como segunda lección cabe destacar que en dicha orientación hay cabida tanto para la simple indicación como para la exhortación (sea prescriptiva o prohibitiva). La cuestión no es si se debe orientar o no, sino que cómo hacerlo.

\footnotetext{
${ }^{24}$ Por problemas de espacio, se ofrecen otros casos en esta nota de pie de página. Estos son: prescriptive, OxDL (s.v.), presriptif, DLFr (s.v.), pescriptive grammar, DLL (s.v.); usage, DLP (s.v.), DLFr (s.v.), Sprachgebrauch, MLSp, (s.v.); archaism, DLP (s.v.), DLL (s.v.), archaïsme, DLaL (s.v.), Archaismus MLSp, (s.v.)) orthoepy, DLP (s.v.), orthoépie, DLaL (s.v.); orthographe, DLaL (s.v.), DLFr (s.v.), Orthographie, MLSp (s.v.).
} 
Es cometido de la lingüística elaborar los instrumentos teóricos y metodológicos que permitan ejecutar esta tarea. No cabe duda de que en este sentido la lingüística hispánica se encuentra en un alto estadio de desarrollo.

En un ámbito más restringido, le compete también a la lexicografía hacerse cargo de la orientación. El hablante va al diccionario de lengua justamente porque vacila o duda frente a un determinado fenómeno. Es función del diccionario orientarlo debidamente, ya sea comentando un uso, ya sea exhortándolo a usar o no una dada forma.

\section{Bibliografías}

Abdi, Heidar y Asidi, Bahareh. “A Synopsis of Researches on Teachers' and Students' Beliefs about Language Learning”. IJSELL, 3-4 (2015): 104-114. https://www.arcjournals.org/pdfs/ijsell/v3-i4/14.pdf.

Abraham, Werner. Terminologie zur neueren Linguistik. Tübingen: Niemeyer, 1974.

Bechara, Evanildo. Novo Dicionário de Dúvidas da Língua Portuguesa. Rio de Janeiro: Nova Fronteira, 2016.

Baldinger, Kurt. Teoría semántica. Hacia una semántica moderna. Madrid: Alcalá, 1977.

Blackburn, Simon. Dicionário Oxford de Filosofia. Rio de Janeiro: Jorge Zahar, 1997.

Boysson-Bardies, Bénédicte. ¿Qué es el lenguaje? México D.F. : FCE, 2007.

Bußmann, Hadumod. Routledge Dictionary of Language and Linguistics. London: Routledge, 2006. Diccionarios evaluados

Coseriu, Eugenio. Einführung in die Allgemeine Sprachwissenschaft. Tübingen: Francke, 1992.

Crystal, David. English as a Global Language. Cambridge: CUP, 2003.

Crystal, David. The Cambridge Encyclopedia of Language. Cambridge: CUP, 2003.

Crystal, David. A Dictionary of Linguistics and Phonetics. Malden / Oxford / Victoria: Blackwell, 2008. Diccionarios evaluados

Dubois, Jean et alii. Dictionnaire de Linguistique et des Sciences du Langage. Paris: Larousse, 2013. Diccionarios evaluados

Elizaincín, Adolfo. "Un importante reto para la política lingüística panhispánica". Actas del VII Congreso Internacional de la Lengua Española. Puerto Rico, 2016. http://congresosdelalengua.es/puertorico/ponencias/seccion_5/ponencias_seccio N5/elizaincin-adolfo.htm.

Eckkrammer, Eva Martha y Lescure, Stéphanie. "Aménagement linguistique et défense istitutionnalisée de la langue : France". Manuel de linguistique française. Eds. Claudia Polzin-Haumann y Wolfgang Schweickard. Gruyter, 2015. 133-159.

Engelberg, Stefan y Lemnitzer, Lothar. Lexikographie und Wörterbuchbenutzung. Tübingen: Stauffenburg, 2004.

Farias, Virginia. "La política panhispánica y su reflejo en las obras académicas. Consideraciones acerca del tratamiento de la variación diatópica y diastráticodiafásica en el DRAE". Romaniae Pontes. Beiträge zur Geschichte der Gallound Iberoromania. Hrsg. Anna-Susan Franke y Vicente Àlvarez-Vives. Berlin: Peter Lang, 2018. 161-175.

Glück, Helmut y Werner Sauer, Wolfgang. Gegenwartsdeutsch. Stuttgart: J.B. 
Metzlersche Verlagsbuchhandlung, 1990.

Glück, Helmut (Hrsg.). Metzler Lexikon Sprache. Stuttgart: Metzler, 2010.

Matthews, P.H. Oxford Concise Dictionary of Linguistics. Oxford: OUP, 2014. Diccionarios evaluados

Hartmann, R.R.K y James, Gregory. Dictionary of Lexicography. London: Routledge, 2001.

Henne, Helmut. "Die Berufung wird stattgegeben. Plädoyer für die Entwicklung von Sprachgefühl“. Hans-Martin Gauger, Henne Helmut, Wulf Oesterreicher, Manfred Geiier y Wolfgang Müller. Oesterreicher,lf Sprachgefühl? Vier Antworten auf eine Preisfrage. Heidelberg: Norbert Lambert, 1982. 91-137.

Jackobson, Roman. "Zwei Seiten der Sprache und zwei Typen aphasischer Störungen". Aufsätze zur Linguistik und Poetik. Jackobson, Roman. Frankfurt am Main: Ullstein, 1979. 117-141.

Kirchner, Friederich.. Wörterbuch der philosophischen Begriffe. Hamburg: Felix Meiner, 2013.

Kunkel-Razum, Kathrin, Holzwarth-Raether, Ulrike, Gallmann, Peter y Klaußner, Burghart. Warum es nicht egal ist, wie wir schreiben. Berlin: Bibliographisches Institut 2018.

Lázaro Carreter, Fernando. Diccionario de términos filológicos. Madrid: Gredos, 1981. Maldonado Cárdenas, Mireya. "El carácter pluricéntrico del español y sus variedades". Romaniae Pontes. Beiträge zur Geschichte der Gallo- und Iberoromania Hrsg. Anna-Susan Franke y Vicente Àlvarez-Vives. Berlin: Peter Lang, 2018. 435-446.

Martínez de Souza, José. Diccionario de Lexicografía Práctica. Barcelona: Bibliograf, 1995.

Morales Pettorino, Félix. "Nuestro dialecto". El español de Chile. Estudios fónicos, gramaticales y léxicos. Félix Morales Pettorino. Valparaíso: Editorial Puntángeles, 2007. 35-60.

Moreno de Alba, José. El español en América. México. D.F.: Fondo de Cultura Económica, 1995.

Moreno Fernández, Francisco. "La búsqueda de un español global". Actas del VII Congreso Internacional de la Lengua Española. Puerto Rico, 2016. http://congresosdelalengua.es/puertorico/ponencias/seccion_5/ponencias_seccio N5/moreno-francisco.htm.

Mugglestone, Lynda. Dictionaries. A Very Short Introduction. Oxford: OUP, 2011.

Nunan, David. "The Impact of English as a Global language on Educational Policies and Practices the Asia-Pacific Region". TESOL Quarterly, 37.4 (2003): 589613.

Peters, Pam. "Prescription and description". The Handbook of English Linguistics. Eds. Aarts, Bas y April McMahon. Malden, Oxford, Victoria: Blackwell Publishing, 2006. 759-780.

Piller, I. “Language ideologies”. In: K. Tracy, C. Ilie, \& T. Sandel (eds.), The International Encyclopedia of Language and Social Interaction. West Sussex: Wiley-Blackwell, vol. 2. 2015, 917-927.

Rabanales, Ambrosio. “Qué es hablar correctamente?" Revista de Educación, 119 (1984): 49-58.

Robins, R.H. A Short History of Linguistics. London: Longman, 1990.

Saussure de, Ferdinand. Cours de Linguistique Générale. Genève: Arbre d'Or, 2005. https://arbredor.com/ebooks/CoursLinguistique.pdf.

Sinclair, John. Corpus, Concordance, Collocation. Oxford: OUP, 1991. 
Tournier, Nicole; Tournier, Jean. Dictionnaire de Lexicologie Française. Paris : Ellipses Édition, 2017. Diccionarios evaluados.

Walter, Henriette. Le français dans tout les sens. Paris: Robert Laffont, 1988.

Wee, Lionel. "Language policy and planning". The Routledge Handbook of Applied Linguistics. Ed. James Simpson. London- New York: Routledge, 2011. 12-23.

Zanatta, Flávia. A normatividade e seu reflexo em dicionários semasiológicos de língua portuguesa. Tesina de maestría. Programa de Pós-Graduação em Letras, Univeridade Federal do Rio Grande do Sul. Porto Alegre, 2010. http://hdl.handle.net/10183/25429.

Zimmermann, Thomas y Sternfeld, Wolfgang. Introduction to Semantics. An Essential Guide to the Composition of Meaning. Berlin: de Gruyter, 2013.

Zolin-Vesz, Fernando. "Crenças sobre o ensino-aprendizagem de espanhol em uma escola pública”. Revista Brasileira de Linguística Aplicada. 13.3 (2013): 815828.

Tournier, Nicole; Tournier, Jean. Dictionnaire de Lexicologie Française. Paris : Ellipses Édition, 2017. Diccionarios evaluados 